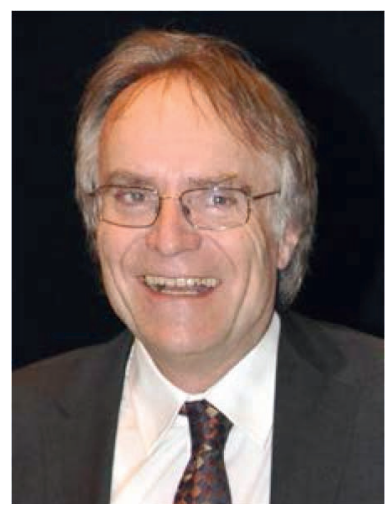

Prof. Dr. sc. nat. Beat Meier, Wädenswil, Schweiz

\section{Phytotherapie im Fokus: Internationale Tagung in Winterthur}

Die Schweizerische Medizinische Gesellschaft für Phytotherapie (SMGP) darf vom 18. bis 21. Juni 2014 die Anwender der Phytotherapie an Mensch und Tier sowie Forschende, die sich mit Arzneipflanzen beschäftigen, und Vertreterinnen und Vertreter der Behörden, die in Deutschland, Österreich und der Schweiz über die Zulassung von pflanzlichen Arzneimitteln entscheiden, in Winterthur begrüssen. Ebenso willkommen heisst sie die wachsende Anzahl an Medizinalpersonen, die sich vom Potenzial der Phytotherapie überzeugen lassen wollen, nachdem Kolleginnen und Kollegen oder die Literatur von positiven Erfahrungen berichtet haben. Zusammen mit der European Scientific Cooperation of Phytotherapy (ESCOP) und der Gesellschaft für Arzneipflanzen- und Naturstoffforschung (GA) zeichnen die drei deutschsprachigen Phytotherapiegesellschaften alle 2 Jahre für diesen gemeinsamen Kongress verantwortlich. Im Juni in Winterthur dürfen sie zudem auf die Unterstützung des Institutes für komplementäre und integrative Medizin, bisher Institut für Naturheilkunde, am Universitätsspital Zürich, des Institutes für Biotechnologie, insbesondere der Fachgruppe Phytopharmazie der Zürcher Hochschule für Angewandte Wissenschaften (ZHAW), und des Institutes für Biologischen Landbau (FiBL) in Frick zählen. Der Anlass findet erstmals in der Schweiz statt und beschliesst so den ersten Zyklus. Das zunehmende Interesse belegen die wachsende Zahl der Teilnehmenden sowie die 64 gemeldeten Posterbeiträge, die neben pharmaziehistorischen
Aspekten die Qualität und Zusammensetzung, die Pharmakologie, die Therapie, die Sicherheit und die Zulassung pflanzlicher Arzneimittel im Fokus haben. Die Poster beleuchten damit verschiedenste Themen, so etwa - neben Wirksamkeitsnachweis und Qualität - die Phytotherapie in Leitlinien, in anerkannten Monographien beschriebene Nebenwirkungen, die unter Einbezug evidenzbasierter Kriterien nicht länger aufrechterhalten werden können, und unter den zahlreichen veterinärmedizinischen Beiträgen die erfolgreiche Anwendung von Johanniskraut bei einem Schimpansen.

Ziel der Veranstaltung und der Gesellschaften ist es, die Phytotherapie und ihr Potenzial bekannter zu machen. Für die Tagung in Winterthur wurde das Thema «Phytotherapie in der Geriatrie» ausgewählt. Die Geriatrie birgt ein für die Phytotherapie auszudehnendes Tätigkeitsgebiet, werden doch vermehrt synthetische Arzneimittel kritisch betrachtet. Dem stehen das geringfügige Interaktionspotenzial und die grosse therapeutische Breite der meisten pflanzlichen Arzneimittel gegenüber. Die vielfach beobachtete Multimedikation mit synthetisch hergestellten Substanzen birgt Risiken, die mit pflanzlichen Arzneimitteln reduziert werden können. Die Vielstoffsysteme weisen in den meisten Fällen keine hochkonzentrierten Inhaltsstoffe auf, weshalb bisher nur sehr vereinzelt relevante Interaktionen bekannt geworden sind. Positive In-vitro-/In-vivo-Korrelationen sind selten, und entsprechend vorsichtig sind oft schlagzeilenträchtige In-vitroPublikationen zu bewerten. Im pharmakologischen Test blockieren Extrakte die Rezeptoren oft nur teilweise, was auf eine regulierende Wirkung hinweist. Von diesem Wissen gilt es zu profitieren. Arzneipflanzen eignen sich jedoch nicht nur in Form von Arzneimitteln als Therapieoptionen, sondern auch als Organismen, die angebaut, gehegt und gepflegt werden können. In vielen Heimen gehört heute das Gärtnern in speziell angelegten Hochbeeten unter Anleitung von Gartentherapeuten zum Grundprogramm. Die Phytotherapie versteht sich als Bestandteil einer modernen Medizin, die über die reine Medikation hinausgeht und in umfassenden, oft individuellen Therapiekonzepten ihren Platz hat. So auch beim alternden und alten Menschen.

Die Tagung will sich zudem der Zukunft widmen. Dafür braucht es Forschung, und so wird das Thema der 28. Schweizerischen Tagung für Phytotherapie vom 21. November 2013 (siehe auch Nachbericht in Ausgabe 2/2014 der SchweIZerischen Zeitschrift für GanZHEITSMEDIZIN) fortgesetzt. Metanalysen und Versorgungs-

\section{KARGER}

Fax +497614520714 Information@Karger.com www.karger.com
Prof. Dr. sc. nat. Beat Meier

Dozent für Phytopharmazie, Geschäftsleiter SMGP

Zürcher Hochschule für Angewandte Wissenschaften

Postfach, 8260 Wädenswil, Schweiz

beat.meier@zhaw.ch 
forschung werden bzw. sind schon wichtige Instrumente für den Nachweis von Wirksamkeit, Zweckmässigkeit und Wirtschaftlichkeit von Therapien. Die Phytotherapie will sich diesen Herausforderungen stellen. Entsprechende Konzepte wurden und werden entwickelt, auch wenn die Forschungsförderung in den letzten Jahren trotz ausgewiesenem Bedarf in Europa gering geblieben ist. Die Zukunft fordert auch die Zulassungsbehörden: Es müssen Wege gefunden werden, die es wieder attraktiv erscheinen lassen, pflanzliche Arzneimittel zur Zulassung zu bringen. Obwohl die Bereitschaft, pflanzliche Arzneimittel zuzulassen, in den deutschsprachigen Ländern nach wie vor gross und in Europa in den letzten Jahren dank der Etablierung eines Herbal Medicinal Product Committee (HMPC) bei der European Medical Agency (EMA) verankert worden ist, sind die Hürden und damit die Kosten in den letzten Jahren so gewachsen, dass die Hersteller mit Neuentwicklungen zurückhaltend geworden sind und Innovationen selten bleiben. Das Potenzial der neuen HMPC-Monographien wurde bisher nur sehr ungenügend genutzt. Erstmals seit vielen Jahren treten die Verantwortlichen für pflanzliche Arzneimittel der Behörden aus Deutschland, Österreich und der Schweiz wieder gemeinsam an einem Anlass auf - mit Referaten und im Rahmen der Podiumsdiskussion.

Schliesslich soll die Phytotherapie auch in der Veterinärmedizin wieder eine Zukunft haben. Anders als in der
Humanmedizin ist die Phytotherapie dort aus dem Arzneimittelschatz fast vollständig verschwunden, sodass mit Felduntersuchungen und historischen Arbeiten versucht werden muss, altbekannte Therapieerfahrungen wieder zugänglich zu machen. Das veterinärmedizinische Parallelprogramm am Freitag, 20. Juni 2014, wird eine Übersicht über den Wert und den Stand des Erfahrungswissens vermitteln - mit der Hoffnung, dass Phytotherapie in $\mathrm{Zu}$ kunft bei Tieren wieder erfolgreich eingesetzt wird, etwa zur Reduktion der teilweise grossflächig eingesetzten Antibiotika.

Letztlich steht diese Tagung am Schluss meiner Hochschullaufbahn und derjenigen von Professor Dr. Reinhard Saller. Wir werden zwar weiterhin für die Phytotherapie tätig sein, übergeben aber das Institut am Universitätsspital Zürich sowie die Fachgruppe an der ZHAW in jüngere Hände bzw. haben dies bereits getan. Wir sind zuversichtlich, dass die Phytotherapie auch zukünftigen Generationen helfen und weiterhin Gegenstand nutzbringender Forschungstätigkeit sein wird. Wir freuen uns darauf, Sie als Leser und Leserinnen der SCHWEIZERISCHEN ZEITSCHRIFT FÜR GANZHEITSMEDIZIN auch aus diesem Anlass in Winterthur begrüssen zu dürfen (Informationen und Anmeldetool für die Tagung «Phytotherapie 2014: Praxis und Klinik» unter http://phytotherapie2014.smgp.ch). 